\title{
Standard Cosmology from Sigma-Model
}

\author{
Conall Kennedy \\ School of Mathematics, Trinity College, University of Dublin, Ireland
}

\begin{abstract}
We investigate $(4+1)$ - and $(5+0)$-dimensional gravity coupled to a non-compact scalar field sigma-model and a perfect fluid within the context of the Randall-Sundrum scenario. We find cosmological solutions with a rolling fifth radius and a family of warp factors. Included in this family are both the original Randall-Sundrum solution and the self-tuning solution of Kachru, Schulz and Silverstein. Our solutions exhibit conventional cosmology.
\end{abstract}

PACS numbers: 04.50.+h, 11.27.+d, 98.80.Cq.

Keywords: Randall-Sundrum, Domain Walls, Warp Factor, Cosmology, Rolling Dilatons.

*e-mail: conall@maths.tcd.ie

**e-mail: prodanov@maths.tcd.ie 


\section{Introduction}

Theories with extra dimensions where our four-dimensional world is a hypersurface (three-brane) embedded in a higher-dimensional spacetime and at which gravity is localised have been the subject of intense scrutiny since the work of Randall and Sundrum [1]. The main motivation for such models comes from string theory where they are reminiscent of the HoravaWitten solution [2] for the field theory limit of the strongly-coupled $E_{8} \times E_{8}$ heterotic string. The Randall-Sundrum (RS) scenario may be modelled [3] and [4] by coupling gravity to a scalar field and mapping to an equivalent supersymmetric quantum mechanics problem. A static metric is obtained with a warp factor determined by the superpotential. A generalisation to non-static metrics was considered by Binétruy, Deffayet and Langlois who modelled brane matter as a perfect fluid delta-function source in the fivedimensional Einstein equations [5]. However, this resulted in non-standard cosmology in that the square of the Hubble constant on the brane was not proportional to the density of the fluid. Other cosmological aspects of "braneworlds" have been considered in [6].

In this letter we investigate cosmological solutions of five-dimensional gravity coupled to a scalar field sigma-model. In much of the current literature it is assumed that such scalars depend only on the fifth dimension and that the target space metric is of Euclidean signature. By contrast, we consider a non-compact sigma-model and allow the scalars to depend on time as well as the fifth dimension, which we take to be infinite in extent. We also include a

perfect fluid with energy-momentum tensor $\tilde{T}_{\nu}^{\mu}=\operatorname{diag}(-\rho, p, p, p, P)$ and equations of state $P=\omega \rho, p=\tilde{\omega} \rho$. A family of warp factors that includes both the original RS solution and the self-tuning solution of Kachru, Schulz and Silverstein [7] is found. The fifth radius is time-dependent. We find that the fluid exists provided $\omega=\tilde{\omega}=1$. Conventional cosmology is also obtained.

It may appear somewhat unnatural to have an indefinite target space metric since some of the scalars then have "wrongly-signed" kinetic terms. However, such scalars have been considered before in the literature. Within the context of $d+1$ gravity they are descended from vector fields after dimensional reduction along a timelike direction of a higher dimensional "two-time" theory [8] and 49], whilst in $d+0$ dimensions they are interpreted as axions after dualisation of a $(d-1)$-form field strength [10], [11] and [12]. Thus, our paper should be interpreted in the light of these works. 


\section{The Model}

We shall present our calculations in $(4+1)$-dimensional spacetime and only quote analogous results for the $5+0$ case. The action for gravity coupled to two scalars is:

$$
S=\int d^{4} x d r\left(\mathcal{L}_{M A T T E R}^{(5)}+\mathcal{L}_{G R A V I T Y}^{(5)}\right)
$$

where:

$$
\begin{aligned}
\mathcal{L}_{\text {MATTER }}^{(5)} & =-\frac{1}{2} \sqrt{-g^{(5)}} \nabla^{\mu} \phi_{i} \nabla^{\nu} \phi_{j} G^{i j}(\phi) g_{\mu \nu}^{(5)}-\sqrt{-g^{(5)}} U(\phi)-\sqrt{-g^{(4)}} V(\phi) \delta(r), \\
\mathcal{L}_{\text {GRAVITY }}^{(5)} & =\frac{1}{\kappa^{2}} \sqrt{-g^{(5)}} R .
\end{aligned}
$$

Here, $g_{\mu \nu}^{(4)}$ is the pull-back of the five-dimensional metric $g_{\mu \nu}^{(5)}$ to the (thin) domain wall taken to be at $r=0$. The wall is represented by a delta function source with coefficient $V(\phi)$ parametrising its tension. We take $G_{i j}=\operatorname{diag}(1,-1)$. The "correctly-signed" scalar, $\phi^{1}$, may be interpreted as the dilaton and the "wrongly-signed" scalar, $\phi^{2}$, as an axion. (It is possible to consider a non-trivial coupling between the two - for example, $G_{i j}=\operatorname{diag}\left(1,-e^{\sigma \phi^{1}}\right)$ is discussed in [11].)

We assume a separable metric with flat spatial three-sections on the wall:

$$
d s^{2}=-e^{-A(r)} d t^{2}+e^{-A(r)} g(t)\left(d x^{2}+d y^{2}+d z^{2}\right)+f(t) d r^{2} .
$$

This is a natural generalisation of the $4 d$ flat Robertson-Walker metric to a RS context.

Given the above ansatz, it is not unreasonable to assume scalars of the form

$$
\phi^{i}(t, r)=a^{i} \psi(t)+b^{i} \chi(r) .
$$

Since $\phi^{i}$ can be considered as coordinates on the target spacetime we must require them to be linearly independent. This imposes the condition

$$
\operatorname{det}\left(\begin{array}{ll}
a^{1} & b^{1} \\
a^{2} & b^{2}
\end{array}\right) \neq 0 .
$$

The Schwarz inequality $\frac{(a \cdot a)(b \cdot b)}{(a \cdot b)^{2}}<1$ follows as a corollary.

We also make the ansatz that both the potentials $U$ and $V$ are of Liouville type (see, for instance, [13]):

$$
\begin{aligned}
V(\phi) & =V_{0} e^{\alpha_{i} \phi^{i}}, \\
U(\phi) & =U_{0} e^{\beta_{i} \phi^{i}} .
\end{aligned}
$$


The energy-momentum tensor for the scalar fields is:

$$
\begin{aligned}
& T_{\mu \nu}^{(0)}=\frac{1}{2} \nabla_{\mu} \phi^{i} \nabla_{\nu} \phi^{j} G_{i j}-\frac{1}{2} g_{\mu \nu}\left(\frac{1}{2} \nabla_{\alpha} \phi^{i} \nabla_{\beta} \phi^{j} G_{i j} g^{\alpha \beta}+U(\phi)\right) \\
& -\frac{1}{2} \frac{\sqrt{-g^{(4)}}}{\sqrt{-g^{(5)}}} V(\phi) \delta(r) g_{a b}^{(4)} \delta_{\mu}^{a} \delta_{\nu}^{b}
\end{aligned}
$$

We introduce a perfect fluid via its energy-momentum tensor:

$$
\tilde{T}_{\nu}^{\mu}=\operatorname{diag}(-\rho, p, p, p, P)
$$

with $\rho$ the density and $p$ and $P$ the pressures in the $x, y, z$ and fifth dimensions respectively. The preferred coordinate system (3) is taken as the rest frame of the fluid.

Einstein's equations $G_{\mu \nu}=\kappa^{2}\left(T_{\mu \nu}^{(0)}+\tilde{T}_{\mu \nu}\right)$ reduce to:

$$
\begin{aligned}
& \frac{1}{4} \frac{\dot{f}}{f} \frac{\dot{g}}{g}+\frac{\dot{g}^{2}}{g^{2}}+\frac{1}{4} \frac{\dot{f}^{2}}{f^{2}}-\frac{1}{2} \frac{\ddot{f}}{f}-\frac{\ddot{g}}{g}-\frac{\kappa^{2}}{2} a \cdot a \dot{\psi}^{2}-\kappa^{2} e^{-A}(\rho+p)=0 \\
& \frac{3}{4} \frac{\dot{f}}{f} \frac{\dot{g}}{g}+\frac{3}{4} \frac{\dot{g}^{2}}{g^{2}}-\frac{\kappa^{2}}{4} a \cdot a \dot{\psi}^{2}-\kappa^{2} e^{-A} \rho=0 \\
& \frac{3}{2}\left(A^{\prime 2}-A^{\prime \prime}\right)+\frac{\kappa^{2}}{4} b \cdot b \chi^{\prime 2}+\frac{\kappa^{2}}{2} f U+\frac{\kappa^{2}}{2} f^{1 / 2} V \delta(r)=0 \\
& \frac{3}{2} \frac{\ddot{g}}{g}+\frac{\kappa^{2}}{4} a \cdot a \dot{\psi}^{2}+\kappa^{2} e^{-A} P=0 \\
& \frac{3}{2} A^{\prime 2}-\frac{\kappa^{2}}{4} b \cdot b \chi^{\prime 2}+\frac{\kappa^{2}}{2} f U=0 \\
& \frac{3}{2} A^{\prime} \frac{\dot{f}}{f}+\kappa^{2} a \cdot b \dot{\psi} \chi^{\prime}=0 .
\end{aligned}
$$

In the above equations we have assumed separability. This requires that the density and pressures are each of the form $e^{A(r)}$ times a function of $t$. We are interested in solutions with $\dot{f} \neq 0$. This requires $a \cdot b \neq 0$, as can be deduced from (14).

The equations of motion for the scalar fields

$$
\nabla^{2} \phi^{j} G_{j k}-\frac{\partial U(\phi)}{\partial \phi^{k}}-\frac{\sqrt{-g^{(4)}}}{\sqrt{-g^{(5)}}} \frac{\partial V(\phi)}{\partial \phi^{k}} \delta(r)=0
$$


result in the following bulk equations

$$
\begin{aligned}
\partial_{t}\left(f^{1 / 2} g^{3 / 2} \dot{\psi}\right) & =0, \\
b_{i}\left(2 A^{\prime} \chi^{\prime}-\chi^{\prime \prime}\right)+f \beta_{i} U_{0} & =0,
\end{aligned}
$$

and the jump condition:

$$
\lim _{\epsilon \rightarrow 0^{+}}\left[b_{i}\left(\chi^{\prime}(\epsilon)-\chi^{\prime}(-\epsilon)\right)\right]=\alpha_{i} f^{1 / 2} V(\phi(t, 0)) .
$$

\section{The Solutions}

Equation (14) implies that we can make the following choice:

$$
\begin{aligned}
\kappa \chi^{\prime}(r) & =\sqrt{6} A^{\prime}(r), \\
\kappa \dot{\psi}(t) & =-\frac{\sqrt{6}}{4} \frac{1}{a \cdot b} \frac{\dot{f}(t)}{f(t)} .
\end{aligned}
$$

The Warp Factor

Inserting (19) into (13) gives $U(\phi)$ as:

$$
U=-\frac{3}{\kappa^{2}} \frac{1}{f} A^{\prime 2}(1-b \cdot b) .
$$

We can express the domain wall potential $V(\phi) \delta(r)$ as $V(\phi) \delta(r)=$ $V_{0} f(t)^{-1 / 2} \delta(r)$. Equation (11) can then be rewritten in the form

$$
A^{\prime \prime}-2 b \cdot b A^{2}-\frac{\kappa^{2}}{3} V_{0} \delta(r)=0,
$$

yielding the following options for $A(r)$ and $V_{0}$ :

1. If $b \cdot b=0$, we find $A(r)=2 \sigma k|r|$, where $\sigma= \pm 1$. Then $V_{0}=12 \sigma k \kappa^{-2}$. $\sigma=-1$ is the RS1 solution and $\sigma=+1$ is the RS2 solution, as described in [14].

2. If $b \cdot b \neq 0$, we find $A(r)=\xi \ln (k|r|+1)$ where $\xi=-\frac{1}{2 b \cdot b}$ and $V_{0}=-\frac{3 k \kappa^{2}}{b \cdot b}$. If $b \cdot b$ and $k$ are both positive, then this represents the selftuning solution of Kachru, Schulz and Silverstein [7]. As observed in [15] and [16], if $k<0$ there are naked singularities at $|r|=-1 / k$ whose interpretation is currently of some debate [17].

The above forms for $U$ and $V$ are consistent with (6) if $\alpha_{i}=\frac{\beta_{i}}{2}=\frac{2 \kappa b_{i}}{\sqrt{6}}$ and $U_{0}=-\frac{3}{\kappa^{2}} A^{\prime 2}(0)(1-b \cdot b)$. It can now be verified that (17) is equivalent to (22) in the bulk, whilst (18) yields no further information. 


\section{The Cosmology}

The equation of motion (16) implies that

$$
\dot{\psi}(t)=\frac{1}{\kappa} f(t)^{-1 / 2} g(t)^{-3 / 2} .
$$

This assumes $f$ is not constant, otherwise (16) is trivially satisfied due to (20). We find that $f(t)$ and $g(t)$ are related via the following equation:

$$
\frac{\dot{f}(t)}{f(t)^{1 / 2}}=\mu g(t)^{-3 / 2}
$$

where $\mu=-\frac{4 a \cdot b}{\sqrt{6}}$.

Adding equations (10) and (12) gives:

$$
\dot{g}^{2}+2 g \ddot{g}+\frac{\dot{f}}{f} \dot{g} g+\frac{4}{3} \kappa^{2} g^{2} e^{-A}(P-\rho)=0 .
$$

On the otherhand, using (10) and (16) in (9) we obtain:

$$
\dot{g}^{2}+2 g \ddot{g}+\frac{\dot{f}}{f} \dot{g} g+2 \kappa^{2} g^{2} e^{-A}(p-\rho)=0 .
$$

Consequently, the relation

$$
p=\frac{1}{3} \rho+\frac{2}{3} P,
$$

may be deduced.

We now assume the equation of state $P=\omega \rho$ or, equivalently, $p=\frac{1}{3}(1+$ $2 \omega) \rho \equiv \tilde{\omega} \rho$. From (10) and (20), the density $\rho$ is given by

$$
\rho(t, r)=\frac{3 e^{A}}{4 \kappa^{2}}\left(\frac{\dot{f}}{f} \frac{\dot{g}}{g}+\frac{\dot{g}^{2}}{g^{2}}-\frac{a \cdot a}{8(a \cdot b)^{2}} \frac{\dot{f}^{2}}{f^{2}}\right),
$$

so that (25) may be alternatively expressed as:

$$
\omega \frac{\dot{g}^{2}}{g^{2}}+2 \frac{\ddot{g}}{g}+\omega \frac{\dot{f}}{f} \frac{\dot{g}}{g}+(1-\omega) \frac{a \cdot a}{8(a \cdot b)^{2}} \frac{\dot{f}^{2}}{f^{2}}=0 .
$$

Taken together with (24), equation (29) defines the cosmology.

We seek either power law, $f \sim t^{q}$, or exponential (inflationary), $f \sim e^{\gamma t}$, solutions of (29). The corresponding solutions for $g(t)$ are $g \sim t^{(2-q) / 3}$ and $g \sim e^{-\gamma t / 3}$ respectively. The exponents $q$ and $\gamma$ are non-zero but otherwise 
arbitrary.

There are two cases to consider: $\omega=1$ and $\omega \neq 1$.

(A) $\omega=1$

From (28), it follows that in the exponential case the density is positive provided $\frac{a \cdot a}{(a \cdot b)^{2}}<-\frac{16}{9}$ (independently of $\gamma$ ). On the otherhand, the density is positive in the power law case provided $\frac{a \cdot a}{(a \cdot b)^{2}}<h(q) \equiv \frac{16}{9} \frac{(2-q)(1+q)}{q^{2}}$. As shown in the figure below, the minimum of $h(q)$ is -2 so we can achieve

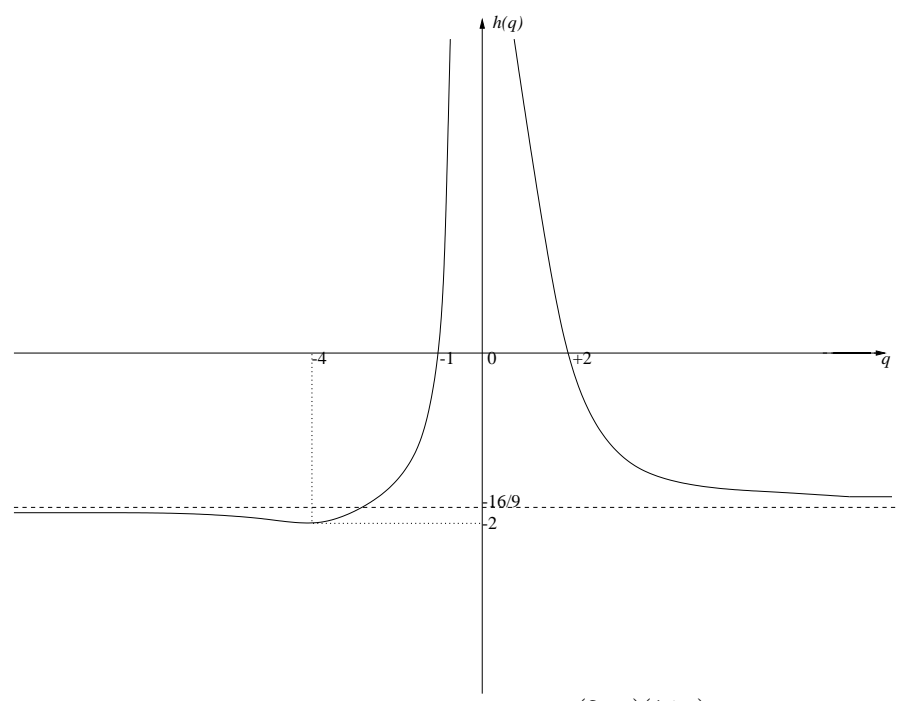

Figure: A sketch of the function $h(q)=\frac{16}{9} \frac{(2-q)(1+q)}{q^{2}}$. The function has a minimum of -2 at $q=-4$ and tends to $-\frac{16}{9}$ as $q \rightarrow \pm \infty$.

positive density for all $\gamma$ and $q$ if we choose $\frac{a \cdot a}{(a \cdot b)^{2}}<-2$ Ð. Defining the scale factor and Hubble constant as per usual by $a^{2}(t)=g(t)$ and $H=\dot{a} / a$, it is easy to see that we obtain conventional cosmology, $H^{2} \propto \rho$, for both the power law and exponential cases.

(B) $\omega \neq 1$

Solution of (29) leads to the above inequalities for $\frac{a \cdot a}{(a \cdot b)^{2}}$ becoming strict equalities which, in turn, leads to the vanishing of the density. Hence, the fluid only exists if $\omega=1$.

\section{The Euclidean Case}

The only essential difference between the $5+0$ case and the $4+1$ case considered above is that $\tilde{T}_{\nu}^{\mu}$ flips sign. This changes the sign of $\rho$ in $(28)$ so that

\footnotetext{
${ }^{1}$ This choice is consistent with the Schwarz inequality provided $b \cdot b>-\frac{1}{2}$. If $b \cdot b \leq-\frac{1}{2}$, positive density is achieved only for a limited range of $q$.
} 
the density is positive if $\frac{a \cdot a}{(a \cdot b)^{2}}>-2$. Similar considerations (see previous footnote) apply as to the range of $q$.

\section{Discussion}

We note in passing that the scalar field equations of motion, (15), imply that $\nabla^{\mu} T_{\mu \nu}^{(0)}=0$ (and conversely off the brane only). This, in turn, implies that the fluid equation of motion $\nabla_{\mu} \tilde{T}_{\nu}^{\mu}=0$ is automatically satisfied. In this sense, the same results in the bulk can be obtained from Einstein's equations and $\nabla_{\mu} \tilde{T}_{\nu}^{\mu}=0$.

It may seem a bit unusual to consider a non-static fifth radius (some authors [19 give arguments against rolling dilatons). We would like to present an intuitive argument in favour of our choice. Consider a five-dimensional spacetime with Robertson-Walker metric:

$$
d s^{2}=-d t^{2}+g(t)\left(d x^{2}+d y^{2}+d z^{2}+d R^{2}\right) .
$$

The $(x, y, z, R)$-space is isotropic. Change coordinates via

$$
d r=e^{-\frac{1}{2} A(r)} d R
$$

and perform a conformal transformation of the metric:

$$
d s^{2} \rightarrow e^{-A(r)} d s^{2} .
$$

Then the metric becomes:

$$
d s^{2}=-e^{-A(r)} d t^{2}+e^{-A(r)} g(t)\left(d x^{2}+d y^{2}+d z^{2}\right)+g(t) d r^{2}
$$

The warp factor of the conformal transformation violates the symmetry between the four spatial coordinates. Zel'dovich [20] gives arguments that any universe will become isotropic with time and non-isotropic expansion causes particle creation. To avoid particle creation in the bulk one could restore isotropy by "untwisting" the fifth dimension with another warp factor, i.e., replacing $g(t)$ by another function of time, $f(t)$, such that the four spatial dimensions are still isotropic.

Within our model we can still have scalar fields depending on brane coordinates if we require a static fifth radius. In this case we need to introduce viscosity into the fluid by making $\tilde{T}_{\nu}^{\mu}$ non-diagonall. The sum of the energymomentum tensors of the scalar fields and the fluid should then amount to

\footnotetext{
${ }^{2}$ We are grateful to Brian Dolan for discussions on this point.
} 
a purely diagonal tensor.

From our initial separability assumptions and from equation (28) it is clear that if the warp factor decreases with $r$ then the density grows without limit as we go off the brane and the fluid is smoothly distributed over the entire extra dimension.

Considering a thick brane (in Lorentzian or Riemannian signature) within our model is straightforward. Thickening the brane requires only smearing the delta function in the domain wall potential by expressing it as a limit of some delta-sequence, for example, $\delta_{\nu}(r)=\frac{1}{\pi} \frac{\nu}{1+\nu^{2} r^{2}}$, where $\frac{1}{\nu}$ parametrises the brane thickness.

From (9) - (14) it is evident that under the transformation $f \rightarrow-f$ the potentials $U$ and $V$ change sign but otherwise the analysis is unmodified. Thus one can make the fifth dimension timelike rather than spacelike. Such a possibility was alluded to in [21] and [22].

Finally, it would be interesting to see if our model(s) can be embedded in five-dimensional Lorentzian or Euclidean supergravity, as has recently been done for the minimal Randall-Sundrum model in 4+1 dimensions [23], [24].

\section{Acknowledgements}

We are sincerely grateful to Siddhartha Sen for a suggestion that initiated these investigations and for useful comments. We have benefited from fruitful discussions with Brian Dolan, Petros Florides, David Simms, Charles Nash and Andy Wilkins. C. K. acknowledges the support of Trinity College, Dublin and Enterprise Ireland.

\section{References}

[1] L. Randall and R. Sundrum: A Large Mass Hierarchy from a Small Extra Dimension. Phys. Rev. Lett. 83, 3370-3373 (1999), hep-ph/9905221; L. Randall and R. Sundrum: An Alternative to Compactification. Phys. Rev. Lett. 83, 4690-4693 (1999), hep-th/9906064.

[2] P. Hořava and E. Witten: Heterotic and Type I String Dynamics from Eleven Dimensions. Nucl. Phys. B460, 506-524 (1996), hep-th/9510209; P. Hořava and E. Witten: Eleven-Dimensional Supergravity on a Manifold with Boundary. Nucl. Phys. B475, 94-114 (1996), hep-th/9603142.

[3] O. DeWolfe, D. Z. Freedman, S. S. Gubser and A. Karch: Modelling the Fifth Dimension With Scalars and Gravity. hep-th/9909134. 
[4] C. Csaki, J. Erlich, T. J. Hollowood and Yu. Shirman: Universal Aspects of Gravity Localised on Thick Branes. hep-th/0001033.

[5] P. Binétruy, C. Deffayet and D. Langlois: Non-conventional Cosmology from a Brane-Universe. hep-th/9905012.

[6] N. Kaloper and A. Linde: Inflation and Large Internal Dimensions. Phys. Rev. D59 101303 (1999), hep-th/9811141;

T. Nihei: Inflation in the Five-dimensional Universe With an Orbifold Extra Dimension. Phys. Lett. B465, 81-85 (1999), hep-ph/9905487;

J. M. Cline, C. Grojean and G. Servant: Cosmological Expansion in the Presence of an Extra Dimension. Phys. Rev. Lett. 83, 4245-4247 (1999), hep-ph/9906523;

H. B. Kim and H. D. Kim: Inflation and Gauge Hierarchy in Randall-Sundrum Compactification. Phys. Rev. D61 064003 (2000), hep-th/9909053;

P. Kanti, I. I. Kogan, K. A. Olive and M. Pospelov: Cosmological 3Brane Solutions. Phys. Lett. B468, 31-39 (1999), hep-ph/9909481;

E. E. Flanagan, S.-H. Henry Tye and I. Wasserman: Cosmological Expansion in the Randall-Sundrum Brane World Scenario. hepph/9910498;

U. Ellwanger: Cosmological Evolution in Compactified Hořava-Witten Theory Induced by Matter on the Branes. hep-th/0001126;

R. N. Mohapatra, A. Pérez-Lorenzana and C. A. de S. Pires: Inflation in Models with Large Extra Dimensions Driven by a Bulk Scalar Field. hep-ph/0003089;

M. Brändle, A. Lukas and B. A. Ovrut: Heterotic M-Theory Cosmology in Four and Five Dimensions. hep-th/0003256.

[7] S. Kachru, M. Schulz and E. Silverstein: Self-tuning Flat Domain Walls in 5d Gravity and String Theory. hep-th/0001206.

[8] C. M. Hull and R. R. Khuri: Branes, Times and Dualities. Nucl. Phys. B536, 219-244 (1998), hep-th/9808069.

[9] K. S. Stelle: BPS Branes in Supergravity. Lectures given at the ICTP Summer School, Trieste, Italy, in 1996 and 1997. hep-th/9803116.

[10] E. Cremmer, B. Julia, H. Lü and C. N. Pope: Dualisation of Dualities, I. Nucl. Phys. B523, 73-144 (1998), hep-th/9710119.

[11] E. Cremmer, I. V. Lavrinenko, H. Lü, C. N. Pope, K. S. Stelle and T. A. Tran: Euclidean-Signature Supergravities, Dualities and Instantons. Nucl. Phys. B534, 40-82 (1998), hep-th/9803259. 
[12] S. B. Giddings and A. Strominger: Axion-Induced Topology Change in Quantum Gravity and String Theory. Nucl. Phys. B306, 890-907 (1988).

[13] H. A. Chamblin and H. S. Reall: Dynamic Dilatonic Domain Walls. Nucl. Phys. B562, 133-157 (1999), hep-th/9903225.

[14] C. Csaki, M. Graesser, C. Kolda and J. Terning: Cosmology of One Extra Dimension with Localised Gravity. Phys. Lett. B462, 34-40 (1999), hep-ph/9906513.

[15] D. Youm: Bulk Fields in Dilatonic and Self-Tuning Flat Domain Walls. hep-th/0002147;

D. Youm: Dilatonic p-Branes and Brane Worlds. hep-th/0003174.

[16] C. Gómez, B. Janssen and P. Silva: Dilatonic Randall-Sundrum Theory and Renormalisation Group. hep-th/0002042.

[17] S. S. Gubser: Curvature Singularities: the Good, the Bad, and the Naked. hep-th/0002160;

Z. Kakushadze: Localised (Super)Gravity and the Cosmological Constant. hep-th/0005217.

[18] M. Gremm: Thick Domain Walls and Singular Spaces. hep-th/0002040.

[19] B. A. Campbell and K. A. Olive: Nucleosynthesis and the Time Dependence of Fundamental Couplings. Phys. Lett. B345, 429-434 (1995), hep-ph/9411272.

[20] I. B. Zel'dovich and I. D. Novikov: Relativistic Astrophysics. University of Chicago Press (1983).

[21] J. M. Overduin and P. S. Wesson: Kaluza-Klein Gravity. Phys. Rept. 283, 303-380 (1997), gr-qc/9805018.

[22] M. Chaichian and A. B. Kobakhidze: Mass Hierarchy and Localisation of Gravity in Extra Time. hep-th/0003269.

[23] R. Altendorfer, J. Bagger and D. Nemeschansky: Supersymmetric Randall-Sundrum Scenario. hep-th/0003117.

[24] T. Gherghetta and A. Pomarol: Bulk Fields and Supersymmetry in a Slice of $A d S$. hep-ph/0003129. 